\title{
APRENDIZAJES FORMALES, NO FORMALES E INFORMALES EN LA ERA DIGITAL: CONTRIBUCIONES AL DESARROLLO PROFESIONAL DOCENTE
}

\author{
Alba Souto-Seijo', Iris Estévez ${ }^{2}$, Patricia Romero ${ }^{3}$ y Mercedes González-Sanmamed ${ }^{4}$ \\ 1,2,3,4 Universidade da Coruña, España, ${ }^{1}$ a.souto1@udc.es; ${ }^{2}$ iris.estevezb@udc.es; ${ }^{3}$ patricia.romero.rey@udc.es; \\ ${ }^{4}$ mercedes@udc.es
}

\begin{abstract}
Resumen. Nos encontramos inmersos en la Era Digital, la cual se caracteriza por la rápida obsolescencia del conocimiento. Este hecho contribuye a que los docentes deban formarse permanentemente y deban comprender el aprendizaje como un proceso donde se combinan la formación formal, no formal e informal. El presente trabajo tiene como objetivo determinar cómo los aprendizajes formales, no formales e informales contribuyen al desarrollo profesional del profesorado que imparte docencia en Educación Primaria. Esta investigación, de corte cualitativo, se centra en el estudio de cinco casos de docentes de esta etapa educativa de la provincia de A Coruña (Galicia, España). La técnica de recogida de datos fue la entrevista en profundidad o cualitativa. En lo que respecta al procedimiento, las entrevistas fueron grabadas, transcritas y posteriormente se realizó un análisis de contenido. Los resultados evidencian que los docentes llevan a cabo actividades formativas en todos los contextos; sin embargo, valoran en mayor medida los aprendizajes no formales e informales. El presente estudio pone de manifiesto la importancia de integrar todas las oportunidades formativas que se presentan en los contextos por los que transitamos, puesto que todas ellas contribuyen al desarrollo profesional de los docentes.
\end{abstract}

Palabras clave: Aprendizaje a lo Largo de la Vida; Desarrollo Profesional Docente; Formación Docente.

FORMAL, NON-FORMAL AND INFORMAL LEARNING IN THE DIGITAL AGE: CONTRIBUTIONS TO TEACHER PROFESSIONAL DEVELOPMENT

\begin{abstract}
We are in a Digital Age, which is characterized by the rapid obsolescence of knowledge. This contributes to the fact that teachers must be permanently trained and must understand learning as a process where formal, non-formal and informal training is combined. This paper aims to determine how formal, non-formal and informal learning contributes to the professional development of teachers who teach in Primary Education. This qualitative research focuses on the study of five cases of teachers from this educational stage in A Coruña (Galicia, Spain). The data collection technique was the in-depth or qualitative interview. Regarding the procedure, the interviews were recorded, transcribed and subsequently a content analysis was performed. The results show that teachers carry out training activities in all contexts; however, they value nonformal and informal learning to a greater extent. This study highlights the importance of integrating all the training opportunities that arise in the contexts through which we move since all of them contribute to the professional development of teachers.
\end{abstract}

Keywords: Lifelong Learning; Teacher Professional Development; Teacher Training.

\section{INTRODUCCIÓN}

Nos encontramos inmersos en la Era Digital, denominada así por el papel que juega la tecnología en la sociedad actual. Esta ha impactado en todos los ámbitos de la vida humana y ha transformado nuestros hábitos, la manera en que nos relacionamos con los demás e incluso el modo en que aprendemos. De acuerdo con la UNESCO (2013), las tecnologías digitales (TD) son al mismo tiempo un desafío y una oportunidad. En primer lugar, suponen 
un desafío porque se ha incrementado la velocidad con la que se produce y distribuye el conocimiento, por lo que este se vuelve obsoleto muy rápido (González-Sanmamed, Sangrà, Souto-Seijo, \& Estévez, 2018). Asimismo, esta permanente expansión y renovación del conocimiento ha provocado que la formación inicial recibida por los ciudadanos en general, y los docentes en particular, no sea suficiente y se vean obligados a aprender a lo largo y ancho de la vida, puesto que estos no solo tienen la responsabilidad de aprender, sino la de enseñar en un entorno cambiante (González-Sanmamed, Estévez, Souto-Seijo, \& MuñozCarril, 2020; González-Sanmamed, Souto-Seijo, González, \& Estévez, 2019). Tal y como afirman Souto-Seijo, Estévez, Iglesias e González-Sanmamed (2020) el profesorado debe estar preparado para asumir los retos del día a día y así satisfacer las demandas de la sociedad actual, y es aquí donde las tecnologías proporcionan múltiples oportunidades de aprendizaje (Aguaded \& Cabero, 2014).

Las TD permiten aprender en cualquier momento y lugar, es decir, posibilitan un aprendizaje ubicuo (Cope \& Kalantzis, 2009). Este hecho hace que cualquier espacio sea un escenario de aprendizaje y que, por tanto, este no solo tenga lugar en instituciones educativas regladas (Cabero \& Llorente, 2015; Vázquez-Cano, Fernández, \& López, 2019). Es por ello, que cada vez están cobrando más relevancia los contextos no formales e informales, ya que se han vuelto imprescindibles para aprender en la sociedad cambiante en la que nos ha tocado vivir (Sangrà, Estévez, Iglesias, \& Souto-Seijo, 2019). A continuación, se abordan las diferencias entre los tres tipos de aprendizaje surgidos en dichos contextos (CEDEFOP, 2014; Comisión Europea, 2012; Valdés, Pilz, Rivero, Machado, \& Walder, 2013):

El aprendizaje formal es aquel que tiene lugar en entornos organizados específicamente dedicados a la enseñanza. Este se caracteriza por ser intencional y conlleva a la obtención de un certificado. Un ejemplo de aprendizaje formal es el que se adquiere en las Escuelas Oficiales de Idiomas o en los Centros de Formación y Recursos (CFR), ambos dependientes de la Consellería de Educación, Universidade e Formación Profesional de la Xunta de Galicia.

El aprendizaje no formal se corresponde con el aprendizaje que se lleva a cabo en las diferentes actividades formativas que realizan múltiples instituciones. Este tipo de aprendizaje también es intencional, pero no siempre supone la obtención de un certificado. El aprendizaje de un deporte es un ejemplo de aprendizaje no formal. 
Por último, el aprendizaje informal deriva de la interacción con diferentes personas en situaciones cotidianas, por lo que es fortuito y no implica la obtención de una certificación. Las habilidades sociales adquiridas en actividades de voluntariado sería un ejemplo de aprendizaje informal.

Tal y como afirman Valdés et al. (2013), el aprendizaje formal ha caracterizado el modo de ofrecer formación, por lo que los aprendizajes no formales e informales han sido habitualmente infravalorados. Un ejemplo de ello es la Orden del 14 de mayo de 2013, que regula la convocatoria, el reconocimiento, la certificación y el registro de las actividades de formación permanente del profesorado en Galicia, donde se determina que el reconocimiento de otras actividades formativas desarrolladas en otras entidades tendrá carácter excepcional.

Esta situación debería cambiar, puesto que lo ideal sería integrar todas las oportunidades de formación. De hecho, desde la Comisión Europea se reconoce la importancia de aprovechar y reconocer todas las oportunidades de aprendizaje, ya sean formales, no formales 0 informales (Comisión Europea, 2018), puesto que todas esas oportunidades contribuyen al desarrollo profesional de los docentes y, por tanto, a mejorar la calidad de la enseñanza (Souto-Seijo et al., 2020).

Por todo ello, el presente trabajo se ha llevado a cabo con el objetivo de determinar cómo los aprendizajes formales, no formales e informales contribuyen al desarrollo profesional del profesorado que imparte docencia en la etapa de Educación Primaria.

\section{METODOLOGÍA}

El presente estudio, de corte cualitativo, se ha desarrollado a través de la estrategia del estudio de caso, una de las estrategias más empleadas en educación. Concretamente, este responde a un diseño holístico de múltiples casos (Yin, 2018).

\subsection{Participantes}

A través de un muestreo no probabilístico (Cohen, Manion, \& Morrison, 2018) se seleccionó a cinco docentes que desempeñan su labor en la etapa de Educación Primaria de varios colegios públicos de la provincia de A Coruña (Galicia, España). Tal y como se puede observar en la Figura 1, donde se presentan las características de la muestra, los participantes fueron 4 mujeres y 1 hombre con edades comprendidas entre los 29 y los 51 
años. Cabe destacar que, para garantizar el anonimato y la confidencialidad de los datos, se han empleado nombres diferentes a los reales.

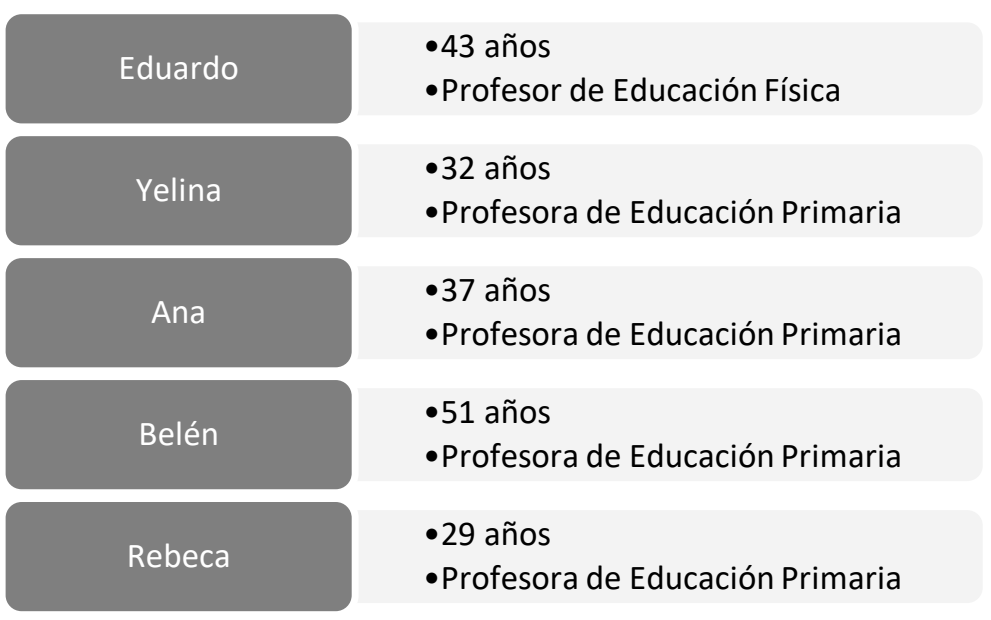

Figura 1. Características de los participantes.

\subsection{Técnica de recogida de datos}

La entrevista en profundidad (Creswell \& Creswell, 2018) ha sido la técnica seleccionada a la hora de recoger la información ya que, como afirma Kvale (2011), permite "explorar la forma en que los sujetos experimentan y entienden su mundo" (p.30). Concretamente, la entrevista tenía como objetivo identificar las actividades que el profesorado lleva a cabo para la mejora de su práctica docente tanto en los contextos formales, como en los no formales e informales. Cabe señalar que previamente, para asegurarse de que se abordaban los temas claves con todos los participantes, se ha elaborado un guión con diferentes preguntas, por lo que ha sido una entrevista semiestructurada (Verd \& Lozares, 2016).

\subsection{Procedimiento}

En primer lugar, se contactó con los docentes seleccionados para explicarles el objetivo de la investigación e invitarlos a participar en la misma. Asimismo, se garantizó el anonimato y la confidencialidad de la información. En ese mismo contacto se resolvieron sus dudas y se acordó con ellos el lugar, día y hora de la entrevista. Es preciso destacar que, para registrar con fidelidad la interacción entre el entrevistador y el entrevistado, se procedió a la grabación de la entrevista. Una vez realizadas las entrevistas, estas fueron transcritas y analizadas. 


\subsection{Análisis de datos}

Tal y como señalan Corbin e Strauss (2014), la interpretación de los datos juega un papel fundamental en investigación cualitativa. Por ello, el análisis se realizó a través del programa Atlas.ti (versión 7), para facilitar la evaluación externa y la discusión de los resultados (Verd \& Lozares, 2016). Concretamente, con el propósito de organizar y obtener significado de la información recopilada, se llevó a cabo un análisis de contenido (Bengtsson, 2016), en el que participaron 2 investigadores. En primer lugar, se realizó una lectura en profundidad de los datos o decodificación y, a continuación, se inició el proceso de codificación de los mismos, mediante el cual se relacionó la información con un código concreto (Saldaña, 2016). A través de este proceso se le otorga sentido a toda la información recopilada, por lo que es fundamental. Con la finalidad de identificar y organizar la información más relevante, y para asegurarse de que el análisis respondía al objetivo de la investigación, se empleó un esquema de análisis, por lo que la codificación ha sido deductiva (Miles, Huberman, \& Saldaña, 2014). Este esquema fue elaborado a partir de la literatura existente y teniendo en cuenta el próposito de la investigación. Así, tal y como se puede observar en la Figura 2, la información fue dividida en dos categorías: aprendizaje formal, y aprendizaje no formal e informal. La primera de ellas hace referencia al aprendizaje que se lleva a cabo de manera estrucutrada en instituciones educativas. La segunda categoría se relaciona con los aprendizajes adquiridos mediante la participación en actividades formativas realizadas por múltiples instituciones (aprendizaje no formal) y en situaciones de la vida cotidiana (aprendizaje informal).

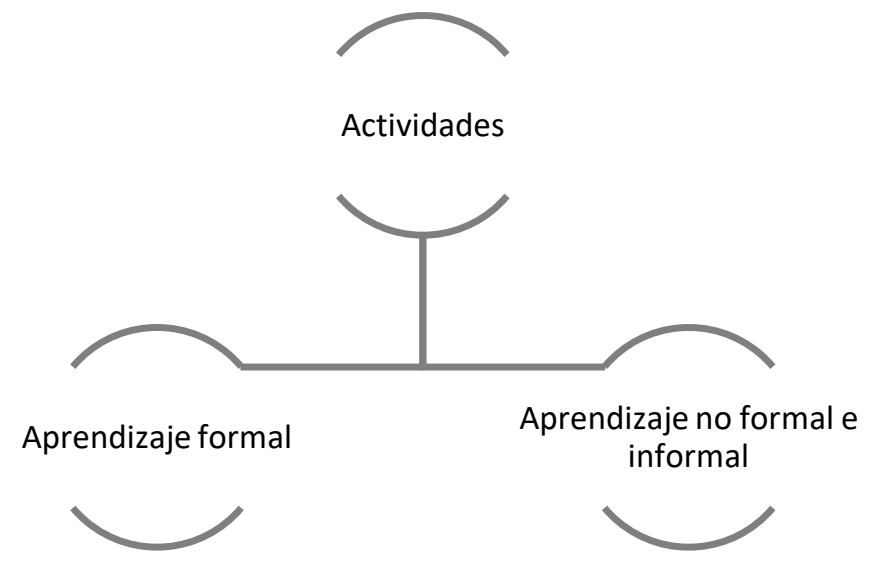

Figura 2. Esquema de análisis.

\section{RESULTADOS}


A continuación, se presentan los resultados del estudio. Estos se han organizado en dos categorías: aprendizaje formal, y aprendizaje no formal e informal.

\subsection{Aprendizaje formal}

En lo que respecta al aprendizaje formal, los cinco participantes en el estudio hacen referencia al aprendizaje adquirido en los cursos que organizan los Centros de Formación y Recursos (CFR). Todos los docentes confirman que participan a lo largo del curso académico en varias de las actividades formativas organizadas por estos centros; y tan solo una de las participantes, Belén, afirma que su participación en estas actividades era mayor al inicio de su carrera como docente: "Una vez que terminé la carrera y empecé a ejercer hice bastantes, porque es cuando te das cuenta de tus necesidades formativas y sobre aquello que necesitas formarte" (E2-B, 3). Esta docente considera que los cursos ofertados son demasiado teóricos, y cree que deberían ser más dinámicos y prácticos.

En general, los cinco docentes reconocen que, aunque la oferta de actividades formales es numerosa, no es suficiente. De hecho, Rebeca piensa que la formación formal no la ayuda a crecer y evolucionar, porque no se alinea con sus motivaciones e inquietudes. Además, Yelina cree que, a pesar que desde los CFR se están haciendo grandes esfuerzos para ofertar actividades formativas adaptadas a las necesidades del profesorado, el abanico de contenidos es limitado: "En mi opinión la formación institucionalizada está siendo cada vez más especializada y adaptada a las necesidades del profesorado, aunque aún queda mucho camino para llegar a una formación 'a la carta' o adaptada a cada profesor" (E2-E, 7). Esta docente, es la única que manifiesta que también acude a la Escuela Oficial de Idiomas y que se ha matriculado en un Máster.

\subsection{Aprendizaje no formal e informal}

En cuanto al aprendizaje no formal e informal, todos los docentes reconocen su importancia. Así, por ejemplo, Eduardo afirma que las actividades de las que derivan este tipo de aprendizajes se suelen desarrollar de forma más dinámica y personalizada que las actividades formativas formales, y esto le anima a participar en ellas habitualmente. De este modo, asiste a jornadas y a congresos, gestiona diversos blogs educativos, participa en un grupo de trabajo relacionado con las tecnologías digitales, colabora en un proyecto de elaboración de materiales educativos digitales y forma parte de un grupo de investigación que trata de mejorar las prácticas educativas. 
Por otro lado, Rebeca y Belén reconocen que a través de Internet se ofertan múltiples y variadas actividades formativas, pero creen que el problema es la calidad. Así, estas docentes afirman que su mayor aprendizaje proviene de las vivencias de su día a día. De hecho, para Rebeca, este aprendizaje es mucho más enriquecedor que el derivado de la formación formal. En este sentido, Belén comenta: "Nunca se deja de aprender, las experiencias vividas ayudan a desarrollar nuevas estrategias que permiten enfrentarse a las siguientes situaciones, así como no cometer los mismos errores. Es decir, se produce aprendizaje, aunque nadie haya previsto que lo hubiera" (E2-B, 8).

Finalmente, Ana asiste habitualmente a encuentros con docentes de la Comunidad Autónoma de Galicia, y Yelina forma parte de diferentes organizaciones y asociaciones de docentes, y es en este contexto donde adquieren la mayor parte de sus aprendizajes no formales e informales.

\section{CONCLUSIONES}

El presente estudio pone de manifiesto la importancia de integrar todas las oportunidades formativas que se presentan en los contextos por los que transitamos, ya sean formales, no formales o informales, puesto que todas ellas contribuyen al desarrollo profesional de los docentes.

Todos los participantes en el estudio le otorgan un gran valor a la formación permanente y creen que es un pilar básico que les ayuda a mejorar su práctica diaria. Estos docentes consideran que la formación inicial que reciben tan solo es la base para después poder seguir formándose a lo largo y ancho de la vida. Tal y como muestran los resultados, los cinco docentes llevan a cabo actividades formativas en todos los contextos, ya que creen que esto les ayuda a desarrollarse profesionalmente e innovar y, por tanto, a mejorar la calidad de la enseñanza. Sin embargo, valoran en mayor medida los aprendizajes no formales e informales. Esto puede ser debido, por una parte, al mayor nivel de especialización, adaptación y flexibilidad que poseen las actividades que conducen a ese tipo de aprendizajes (Colom, 2005) y, por otra, a que la oferta de actividades formativas desarrolladas en los CFR es limitada y, en muchas ocasiones, no responde a sus necesidades e intereses (Sangrà et al., 2019; Souto-Seijo et al., 2020). Aun así, todos ellos participan en estas actividades porque son las que se reconocen en la legislación que regula la formación permanente del profesorado en Galicia. Tal y como se manifestó en la introducción de este estudio, se debe trabajar para que todos los aprendizajes adquiridos 
por los docentes, independientemente del contexto donde hayan sido adquiridos, sean reconocidos y valorados, como bien determina la Comisión Europea (2018). Este reconocimiento contribuirá al desarrollo profesional de los docentes y, por tanto, mejorará la calidad de la enseñanza (Souto-Seijo et al., 2020).

Los resultados obtenidos en esta investigación constituyen un punto de partida para repensar la formación continua de los docentes. Como futura línea de investigación, se propone replicar el estudio en otros contextos para ampliar el tamaño de la muestra. Asimismo, se considera interesante emplear como marco de análisis el concepto de ecologías de aprendizaje, puesto que permite interpretar todas las oportunidades de aprendizaje existentes en el complejo panorama actual, sea cual sea su naturaleza.

Agradecimientos. Este estudio se ha elaborado en el marco del proyecto de investigación ECO4LEARN-HE, financiado por el Ministerio de Economía y Finanzas (Referencia EDU2015-67907R), y gracias a la financiación recibida por una de las autoras de esta investigación, Iris Estévez, en el programa FPI del Ministerio de Economía y Finanzas (BES-2016-077330).

\section{REFERENCIAS}

Aguaded, I., \& Cabero-Almenara, J. (2014). Progress and challenges in promoting educational innovation with emerging and interactive technologies. Educar, 67-83. doi: https://doi.org/10.5565/rev/educar.691.

Bengtsson, M. (2016). How to plan and perform a qualitative study using content analysis. NursingPlus Open, 2, 8-14. doi: https://doi.org/10.1016/j.npls.2016.01.001.

Cabero, J., \& Llorente, M. C. (2015). Tecnologías de la Información y la Comunicación (TIC): escenarios formativos y teorías del aprendizaje. Revista Lasallista de Investigación, 12(2), 186-193. Recuperado de: https://bit.ly/2TpQ4mw.

CEDEFOP (2014). Terminology of European education and training policy. A selection of 130 key terms. Office for Official Publications of the European Communities. Recuperado de: https://bit.ly/2Tmxv2m.

Cohen, L., Manion, L., \& Morrison, K. (2018). Research Methods in Education. London: Routledge.

Colom, A. J. (2005). Continuidad y complementariedad entre la educación formal y no formal. Revista de Educación, 338, 9-22.

Comisión Europea. (2012). Recomendación del Consejo, de 20 de diciembre de 2012, sobre la validación del aprendizaje no formal e informal. Recuperado de: https://bit.ly/2liWAoE.

Comisión Europea. (2018). Recomendación del Consejo, de 22 de mayo de 2018, relativa a las competencias clave para el aprendizaje permanente Texto pertinente a efectos del EEE. Recuperado de: https://bit.ly/38p6wrd.

Cope, B., \& Kalantzis, M. (2009). Ubiquitous Learning. Illinois: University of Illinois Press. Recuperado de: https://bit.ly/2liWYU8.

Corbin, J., \& Strauss, A. (2014). Basics of qualitative research: Techniques and procedures for developing grounded theory. London: Sage Publications. 
Creswell, J. W., \& Creswell, J. D. (2018). Research Design: Qualitative, Quantitative \& Mixed Methods Approaches. London: Sage Publications.

González-Sanmamed, M., Estévez, I., Souto-Seijo, A., \& Muñoz-Carril, P.-C. (2020). Digital learning ecologies and professional development of university professors. Comunicar, 28(62), 9-18. doi: https://doi.org/10.3916/C62-2020-01.

González-Sanmamed, M., Sangrà, A., Souto-Seijo, A., \& Estévez, I. (2018). Ecologías de aprendizaje en la Era Digital: desafíos para la Educación Superior. Publicaciones, 48(1), 25-45. doi: https://doi.org/10.30827/publicaciones.v48i1.7329.

González-Sanmamed, M., Souto-Seijo, A., González, I., \& Estévez, I. (2019). Aprendizaje informal y desarrollo profesional: análisis de las ecologías de aprendizaje del profesorado de educación infantil. Edutec. Revista Electrónica de Tecnología Educativa, (68), 70-81. doi: https://doi.org/10.21556/edutec.2019.68.1305.

Kvale, S. (2011). Las entrevistas en Investigación Cualitativa. Madrid: Ediciones Morata.

Miles, M. B., Huberman, A. M., \& Saldaña, J. (2014). Qualitative Data Analysis. A Methods Sourcebook. London, UK: Sage Publications.

Orden de 14 de mayo (2013). Regula la convocatoria, el reconocimiento, la certificación y el registro de las actividades de formación permanente del profesorado en Galicia. Boletín Oficial del Estado, 96 (22 de mayo de 2013), 17697-17732.

Saldaña, J. (2016). The coding manual for qualitative researchers. London: Sage Publications.

Sangrà, A., Estévez, I., Iglesias, V., \& Souto-Seijo, A. (2019). Desarrollo profesional docente a través de las ecologías de aprendizaje: Perspectivas del profesorado. Edutec. Revista Electrónica de Tecnología Educativa, (68), 42-53. doi: https://doi.org/10.21556/edutec.2019.68.1307.

Souto-Seijo, A., Estévez, I., Iglesias, V., \& González-Sanmamed, V. (2020). Entre lo formal y lo no formal: un análisis desde la formación permanente del profesorado. Educar, 56(1), 97-107. doi: https://doi.org/10.5565/rev/educar.1095.

UNESCO (2013). Enfoques estratégicos sobre las TICS en educación en América Latina y El Caribe. Recuperado de: https://bit.ly/38mGaGf.

Valdés, R., Pilz, D., Rivero, J., Machado, M. M., \& Walder, G. (2013). Aportes conceptuales de la educación de personas jóvenes y adultas: hacia la construcción de sentidos comunes en la diversidad. Recuperado de: https://bit.ly/2uRuzkS.

Vázquez-Cano, E., Fernández, J. M., \& López, E. (2019). Movilidad y ubicuidad en la Educación Superior. In C. Hervás-Gómez, E. Vázquez-Cano, J. M. Fernández-Batanero, \& E. López-Meneses (Eds.), Innovación e investigación sobre el aprendizaje ubicuo y móvil en la Educación Superior (pp. 7-22). Barcelona, España: Octaedro.

Verd, J.M., \& Lozares, C. (2016). Introducción a la investigación cualitativa. Fases, métodos y técnicas. Madrid, España: Síntesis.

Yin, R. K. (2018). Case Study Research and Applications. Design and Methods (6th ed.). London, UK: Sage Publications. 